Publ. RIMS, Kyoto Univ.

18 (1982), 469-474

\title{
Uniqueness of the Infinite Loop Space Structures on Connective Fibre Spaces of BSO
}

\author{
By
}

\section{Akira $\mathrm{KONO}^{*}$}

\section{$\S 1$. Introduction}

In [1] J. F. Adams and S. B. Priddy showed that after localization at any prime $p$ the infinite loop space structure on the space $B S O$ is essentially unique. Let $B O(d, \infty)$ be the $(d-1)$-connected fibre space of $B O$. Then the purpose of the present paper is to show after localizaticn at any prime $p$ the infinite loop space structure on the space $B O(d, \infty)$ is essentially unique if $d \geq 2$. If the word 'localization' is replaced by 'completion', the result continues to hold.

Let $\mathbb{K}_{\boldsymbol{R}}$ be the spectrum which represents classical (periodic) real $K$-theory. Let $d$ be a fixed integer; let $\boldsymbol{b o}(d, \infty)$ be the spectrum obtained from $\mathbb{K}_{\boldsymbol{R}}$ by killing the homotopy groups in degree $<d$, while retaining the homotopy groups in degree $\geq d$. Then $b o(d, \infty)$ represents $(d-1)$-connected real $K$-theory; similarly $\mathbb{K}_{\boldsymbol{C}}$ and $\boldsymbol{b u}(d, \infty)$ in the complex case. Let $\Lambda$ be either the ring $Z_{p}$ of $p$-adic integers or the ring $\mathbb{Z}_{(p)}$ of integers localized at $p$. We can introduce coefficient $A$ into any spectrum $X$ by setting

$$
\mathbb{X}_{\Lambda}=M \Lambda \wedge \mathbb{X}
$$

where $\boldsymbol{M} \Lambda$ is the Moore spectrum for the group $\Lambda$. We write $\mathbb{F}_{p}$ for the field with $p$ elements and $A_{p}$ for the $\bmod p$ Steenrod algebra. Let us arrange for $\boldsymbol{b o}(d, \infty)_{A}$ and $\boldsymbol{b u}(d, \infty)_{A}$ to be $\Omega$-spectra. Note that the equivalence $X_{0} \simeq \Omega X_{1}$ determines an $H$-space structure on $X_{0}$. Then the main purpose of the present paper is to show the following theorem:

Communicated by N. Shimada, August 28, 1981.

* Department of Mathematics, Kyoto University, Kyoto 606, Japan. 
Theorem 1. 1. Let $\boldsymbol{X}=\left\{X_{i}\right\}$ be a connected $\Omega$-spectrum. Suppose given a homotopy equivalence of spaces

$$
X_{0} \simeq B O(d, \infty)_{1},
$$

where $B O(d, \infty)_{\Lambda}$ is the 0 -th term of the $\Omega$-spectrum bo $(d, \infty)_{\Lambda}$. If $d \geq 2$, then there is an equivalence of spectra

$$
\boldsymbol{X} \simeq \boldsymbol{b o}(d, \infty)_{\Lambda} .
$$

The paper is organized as follows:

In Section 2 an $\boldsymbol{Q}$-spectrum $\boldsymbol{\Sigma}^{n} \boldsymbol{X}$ which represents the $n$-fold suspension of $\boldsymbol{X}$ is defined. In the next section the uniqueness of the infinite loop space structures of the space $\Omega S p_{A}$ or the $H$-spaces $\Omega \operatorname{Spin}_{A}$ and $S_{A}$ (definitions are given in Section 3 ) is proved. In Section 4 the main theorem is proved.

Throughout this paper we use the following notation: for a space $X$, $X(n, m)$ denotes that term in the Postnikov system of $X$ whose homotopy groups $\pi_{r}$ are the same as those of $X$ for $n \leq r \leq m$. We use the notation $\boldsymbol{X}(n, m)$ for spectra analogous to that which we use for spaces. The symbol $\underset{H}{\widetilde{T}}$ means $H$-equivalence.

\section{$\S 2$. Suspension of $\boldsymbol{\Omega}$-Spectra}

Let $\boldsymbol{X}=\left\{X_{i}, \varepsilon_{i}: X_{i} \rightarrow \Omega X_{i+1}\right\}$ be an $\Omega$-spectrum and $n$ an integer. Define $Y_{i}$ and $\lambda_{i}$ as follows:

$$
\begin{aligned}
& Y_{i}= \begin{cases}X_{i+n} & i \geq-n \\
\Omega^{-n-i} X_{0} & i<-n,\end{cases} \\
& \lambda_{i}=\left\{\begin{array}{l}
\varepsilon_{i+n}: X_{i+n} \rightarrow \Omega X_{i+n+1} \quad i \geq-n \\
\Omega^{-n-i} 1_{X_{0}}: \Omega^{-n-i} X_{0} \rightarrow \Omega \Omega^{-n-i-1} X_{0} \quad\left(=\Omega^{-n-i} X_{0}\right) \quad i<-n .
\end{array}\right.
\end{aligned}
$$

Then clearly $\boldsymbol{\Sigma}^{n} \boldsymbol{X}=\left\{Y_{i}, \lambda_{i}: Y_{i} \rightarrow \Omega Y_{i+1}\right\}$ is an $\Omega$-spectrum. Moreover $\boldsymbol{\Sigma}^{n} \boldsymbol{X}$ represents the $n$-fold suspension of $\boldsymbol{X}$. The following is easily proved:

Lemma 2. 1. (1) Let $\boldsymbol{X}$ and $\boldsymbol{X}^{\prime}$ be $\Omega$-spectra. Then $\boldsymbol{X}$ is equi- 
valent to $\mathbf{X}^{\prime}$ if and only if $\boldsymbol{\Sigma}^{n} \mathbf{X}$ is equivalent to $\boldsymbol{\Sigma}^{n} \mathbf{X}^{\prime}$ for some $n$.

(2) $H^{*}\left(\boldsymbol{\Sigma}^{n} \boldsymbol{X} ; \boldsymbol{F}_{p}\right)$ is isomorphic to $\Sigma^{n} H^{*}\left(\mathbb{X} ; \mathbb{F}_{p}\right)$ as a module over $A_{p}$ where the graded module $\Sigma^{n} M$ is defined by regarding $M$ so that an element of degree $k$ in $M$ appears as an element of degree $k+n$ in $\Sigma^{n} M$.

(3) $\quad \boldsymbol{\Sigma}^{n}\left(\boldsymbol{\Sigma}^{m} \boldsymbol{X}\right)=\boldsymbol{\Sigma}^{n+m} \boldsymbol{X}$.

\section{§3. Some Postnikov Invariants}

In this section $p=2$ and so $A=A_{2}$ and $\Lambda=\mathbb{Z}_{(2)}$ or $\mathbb{Z}_{2}$. A generator of $H^{n}(\mathbb{E} \mathbb{M}(\Lambda, n) ; \Lambda)$ (resp. $H^{n}(\boldsymbol{E} \boldsymbol{M}(\mathbb{Z} / 2, n) ; \Lambda)$ ) is denoted by $u_{n}$ (resp. $v_{n}$ ) and the mod 2 reduction of $u_{n}$ (resp. $v_{n}$ ) is denoted by $u_{n}^{\prime}$ (resp. $\left.v_{n}^{\prime}\right)$.

First we prove the following:

Lemma 3.1. Let $\mathbb{M}$ be a connected $\Omega$-spectrum such that

$$
M_{0} \simeq \Omega^{6} B O(8, \infty)_{\Lambda}\left(=\Omega S p_{A}\right),
$$

then there is an equivalence of spectra

$$
M \simeq \boldsymbol{\Sigma}^{-6} b \boldsymbol{o}(8, \infty)_{\Lambda} .
$$

Proof. Consider the connected spectrum $M^{\prime}=\boldsymbol{\Sigma}^{-2} M(3, \infty)$, then $M_{0}^{\prime} \underset{H}{\simeq} B O_{\Lambda}$. So $M^{\prime} \simeq \boldsymbol{b o}_{\Lambda}$ by Theorem 1.2 of Adams-Priddy [1]. In particular

$$
H^{*}\left(\boldsymbol{M}^{\prime} ; \mathbb{F}_{2}\right)=H^{*}\left(\boldsymbol{b o} ; \mathbb{F}_{2}\right)=\Sigma^{1}\left(A /\left(A S q^{2}\right)\right) .
$$

Consider the two stage Postnikov system

$$
\boldsymbol{M}(2,3) \rightarrow \boldsymbol{E M}(\Lambda, 2) \stackrel{k}{\longrightarrow} \boldsymbol{E M}(\mathbb{Z} / 2,4) .
$$

Then $k \in H^{4}\left(\boldsymbol{E M}(\Lambda, 2) ; \boldsymbol{F}_{2}\right)=\mathbb{Z} / 2$, which is generated by $S q^{2} u_{2}^{\prime}$. If $k=0$, then $M(2,3)_{0} \simeq K(\Lambda, 2) \times K(\mathbb{Z} / 2,3)$ and so $H^{3}\left(M(2,3)_{0} ; \mathbb{F}_{2}\right) \neq 0$. On the other hand since the natural map $H^{3}\left(M(2,3)_{0} ; \mathbb{F}_{2}\right) \rightarrow H^{3}\left(M_{0} ; \mathbb{F}_{2}\right)$ is a monomorphism and $H^{3}\left(M_{0} ; \mathbb{F}_{2}\right)=H^{3}\left(\Omega S p ; \mathbb{F}_{2}\right)=0$, it follows that $H^{3}\left(M(2,3)_{0} ; \mathbb{F}_{2}\right)=0$. So $k \neq 0$. Then using the $A$-module exact sequence 


$$
0 \rightarrow \Sigma^{4}\left(A /\left(A S q^{2}\right)\right) \underset{\cdot S q^{2}}{\longrightarrow} \Sigma^{2}\left(A /\left(A S q^{1}\right)\right) \rightarrow \Sigma^{2}\left(A /\left(A S q^{1}+A S q^{2}\right)\right) \rightarrow 0,
$$

we have

$$
H^{*}\left(\boldsymbol{M} ; \boldsymbol{F}_{2}\right)=\Sigma^{2}\left(A /\left(A S q^{1}+A S q^{2}\right)\right) .
$$

So by Theorem 1.1 of Adams-Priddy [1], we have $\boldsymbol{\Sigma}^{6} \boldsymbol{M} \simeq \boldsymbol{b} \boldsymbol{o}(8, \infty)_{\Lambda}$ and so $\boldsymbol{M} \simeq \boldsymbol{\Sigma}^{-6} \boldsymbol{b o}(8, \infty)_{1}$.

Next we prove the following:

Lemma 3.2. Let $\boldsymbol{N}$ be a connected $\Omega$-spectrum such that

$$
N_{0} \underset{H}{\simeq} \Omega^{2} B O(4, \infty)_{\Lambda}\left(=\Omega \operatorname{Spin}_{\Lambda}\right),
$$

then there is an equivalence of spectra

$$
\mathbb{N} \simeq \boldsymbol{\Sigma}^{-2} \boldsymbol{b o}(4, \infty)_{\Lambda}
$$

Proof. Consider the connected $\Omega$-spectrum $\boldsymbol{N}^{\prime}=\boldsymbol{\Sigma}^{-4} \boldsymbol{N}(6, \infty)$, then $N_{0}^{\prime} \simeq \Omega^{6} B O(8, \infty)_{\Lambda}$. So $\boldsymbol{N}^{\prime} \simeq \boldsymbol{\Sigma}^{-6} \boldsymbol{b} \boldsymbol{o}(8, \infty)_{\Lambda}$ by Lemma 3.1. In particular,

$$
H^{*}\left(\boldsymbol{N}^{\prime} ; \boldsymbol{F}_{2}\right)=\sum^{2}\left(A /\left(A S q^{1}+A S q^{2}\right)\right) .
$$

Consider the two stage Postnikov system

$$
\mathbb{N}(2,6) \rightarrow \boldsymbol{E M}(\Lambda, 2) \stackrel{k^{\prime}}{\longrightarrow} \boldsymbol{E} \boldsymbol{M}(\Lambda, 7) .
$$

Then $k^{\prime} \in H^{7}(\boldsymbol{E M}(\Lambda, 2) ; \Lambda)=\mathbb{Z} / 2$. Note that since $H^{7}\left(\mathbb{E M}(\Lambda, 2) ; \mathbb{F}_{2}\right)$ $(=\boldsymbol{Z} / 2)$ is generated by $S q^{2} S q^{3} u_{2}^{\prime}, k^{\prime} \neq 0$ if and only if $k^{*}\left(u_{7}^{\prime}\right)=S q^{2} S q^{3} u_{2}^{\prime}$. If $k^{\prime}=0$, then as an infinite loop space $N(2,6)_{0} \simeq K(\Lambda, 2) \times K(\Lambda, 6)$. So as an algebra over the Dyer-Lashof algebra,

$$
H_{*}\left(N(2,6)_{0} ; \boldsymbol{F}_{2}\right)=H_{*}\left(K(\Lambda, 2) ; \boldsymbol{F}_{2}\right) \otimes H_{*}\left(K(\Lambda, 6) ; \mathbb{F}_{2}\right) .
$$

In particular $Q^{4} x_{2}^{\prime}=0$, where $x_{2}^{\prime}$ is a generator of $H_{2}\left(H(2,6)_{0} ; \mathbb{F}_{2}\right)$. On the other hand the natural map $H_{*}\left(N_{0} ; \mathbb{F}_{2}\right) \rightarrow H_{*}\left(N(2,6)_{0} ; \mathbb{F}_{2}\right)$ is isomorphic for $* \leq 6$ and commutes with the Dyer-Lashof operations. But by Theorem 6.9 of Nagata [2], $Q^{4} x_{2} \neq 0$, where $x_{2}$ is a generator of $H_{2}\left(N_{0}\right.$; $\boldsymbol{F}_{2}$ ). So $k^{\prime} \neq 0$. Then using the $A$-module exact sequence

$$
\begin{gathered}
\left.0 \rightarrow \Sigma^{7}\left(A / A S q^{1}+A S q^{2}\right)\right) \cdot \overrightarrow{S q^{2} S q^{3}} \Sigma^{2}\left(A /\left(A S q^{1}\right)\right) \\
\rightarrow \Sigma^{2}\left(A /\left(A S q^{1}+A S q^{2} S q^{3}\right)\right) \rightarrow 0
\end{gathered}
$$


we have

$$
H^{*}\left(\boldsymbol{N} ; \mathbb{F}_{2}\right)=\Sigma^{2}\left(A /\left(A S q^{1}+A S q^{2} S q^{3}\right)\right) \text {. }
$$

So by Theorem 1.1 of Adams-Priddy [1], we have $\boldsymbol{\Sigma}^{2} \mathbb{N} \simeq b \boldsymbol{o}(4, \infty)_{A}$ and so $\boldsymbol{N} \simeq \boldsymbol{\Sigma}^{-2} \boldsymbol{b o}(4, \infty)_{\Lambda}$.

As a corollary of the above lemmas, we can easily show

Corollary 3. 3. Let $\boldsymbol{X}$ be a connected $\Omega$-spectrum such that

$$
\left.X_{0} \simeq B O(4, \infty)_{\Lambda} \text { (resp. } X_{0} \simeq B O(8, \infty)_{1}\right),
$$

then there is an equivalence of spectra

$$
\mathbb{X} \simeq \boldsymbol{b o}(4, \infty)_{\Lambda}\left(\text { resp. } \mathbb{X} \simeq \boldsymbol{b o}(8, \infty)_{\Lambda}\right) .
$$

Proof. If $X_{0} \simeq B O(4, \infty)_{\Lambda}$, then $\left(\Sigma^{-2} X\right)_{0} \underset{H}{\simeq} \Omega^{2} B O(4, \infty)_{\Lambda}$. So by Lemma $3.2, \boldsymbol{\Sigma}^{-2} \boldsymbol{X} \simeq \boldsymbol{\Sigma}^{-2} \boldsymbol{b o}(4, \infty)_{\Lambda}$. The case $X_{0} \simeq B O(8, \infty)_{A}$ is similar.

Using Theorem 6.9 of Nagata [2], we can prove the following by a quite similar method to Lemma 3.2 :

Lemma 3.4. Let $\mathbb{X}$ be a connected $\Omega$-spectrum such that

$$
\chi_{0} \underset{H}{\simeq} \Omega B O(2, \infty)_{\Lambda}\left(=\Omega B S O_{\Lambda}=S O_{\Lambda}\right),
$$

then there is an equivalence of spectra

$$
X \simeq \boldsymbol{Z}^{-1} \boldsymbol{b o}(2, \infty)_{\Lambda}
$$

\section{$\S 4$. Proof of the Main Theorem}

In this section the main theorem, Theorem 1.1 is proved. By the Bott periodicity theorem, $\Omega^{8} B O(n+8, \infty) \simeq B O(n, \infty)$. Then we can easily show

Lemma 4. 1. Let $n, n^{\prime}$ and $n^{\prime \prime}$ be non-negative integers such that $n=8 n^{\prime}+n^{\prime \prime}$. Then $\boldsymbol{\Sigma}^{-8 n^{\prime}} \boldsymbol{b o}(n, \infty) \simeq \mathbb{b o}\left(n^{\prime \prime}, \infty\right)$.

First we assume that $p$ is an odd prime. Then $\Omega^{4} B O(5, \infty)_{A} \simeq$ 
$B O(1, \infty)_{A}$ and

$$
B O(1, \infty)_{A}=B O(2, \infty)_{A}=B O(3, \infty)_{A}=B O(4, \infty)_{A} .
$$

Put $d=4 m+m^{\prime}\left(1 \leq m^{\prime} \leq 4\right)$. Let $\boldsymbol{X}$ be a connected $\Omega$-spectrum such that $X_{0} \simeq B O(d, \infty)_{\Lambda}$. Put $\boldsymbol{X}^{\prime}=\boldsymbol{\Sigma}^{-4 m} \boldsymbol{X}$. Then $X_{0}^{\prime} \simeq B O\left(m^{\prime}, \infty\right)_{\Lambda} \simeq B O_{\Lambda}$. So by Theorem 1.2 of Adams-Priddy [1], we have $\boldsymbol{X}^{\prime} \simeq \boldsymbol{b o}_{\Lambda}\left(\simeq \boldsymbol{b o}\left(m^{\prime}, \infty\right)_{\Lambda}\right.$. Then $\boldsymbol{X} \simeq \boldsymbol{\Sigma}^{4 m} \boldsymbol{b o}\left(m^{\prime}, \infty\right)_{\Lambda} \simeq \boldsymbol{b o}(d, \infty)_{\Lambda}$.

Next we assume $p=2$. Put $d=8 n+n^{\prime} \quad\left(1 \leq n^{\prime} \leq 8\right)$. Note that $B O(1, \infty)=B O, B O(2, \infty)=B S O, B O(3, \infty)=B O(4, \infty)=B$ Spin and $B O(5, \infty)=B O(6, \infty)=B O(7, \infty)=B O(8, \infty)$. So Theorem 1.:1 is true for $d \leq 8$ by Theorem 1.2 of [1] and Corollary 3.3. We may assume that $n \geq 1$. Put $\boldsymbol{X}^{\prime}=\boldsymbol{\Sigma}^{-8 n} \boldsymbol{X}$, then $X_{0}^{\prime} \underset{\boldsymbol{H}}{\widetilde{T}} B O\left(n^{\prime}, \infty\right)_{\Lambda}$. By Theorem 1.1 of [1] and Corollary 3.3, we have $\boldsymbol{X}^{\prime} \simeq \boldsymbol{b o}\left(n^{\prime}, \infty\right)_{\Lambda}$. Then by Lemma 4. 1, we have

$$
\boldsymbol{X} \simeq \boldsymbol{\Sigma}^{8 n} \boldsymbol{b o}\left(n^{\prime}, \infty\right)_{\Lambda} \simeq \boldsymbol{b o}(d, \infty)_{\Lambda} .
$$

For $\boldsymbol{K}_{\boldsymbol{C}}$ we have

Theorem 4.2. Let $\boldsymbol{X}$ be a connected $\Omega$-spectrum. Suppose given a homotopy equivalence of spaces

$$
X_{0} \simeq B U(d, \infty)_{\Lambda} .
$$

If $d \geq 3$, then there is an equivalence of spectra

$$
\boldsymbol{X} \simeq \boldsymbol{b} \boldsymbol{u}(d, \infty)_{\Lambda} .
$$

\section{References}

[1] Adams, J. F., and Priddy, S., Uniqueness of BSO, Math. Proc. Camb. Phil. Soc., 80 (1976), 475-509.

[2] Nagata, M., On the uniqueness of Dyer-Lashof operations on the Bott periodicity spaces, Publ. RIMS, Kyoto Univ., 16 (1980), 499-511. 